\title{
APLICAÇÃO DE HIBRIDISMO NA GESTÃO DE EQUIPES E STAKEHOLDERS DE MÚLTIPLOS PROJETOS DE ENGENHARIA
}

\author{
THE APPLICATION OF HYBRIDISM IN TEAM AND STAKEHOLDER \\ MANAGEMENT AT MULTIPLE ENGINEERING PROJECTS
}

\author{
Tawnee Gomes Chies \\ Mestra em Administração \\ Universidade Nove de Julho - UNINOVE \\ São Paulo, SP - Brasil \\ tawnee.chies@gmail.com \\ Aline Jade Gonçalves Rocha \\ Mestra em Administração \\ Universidade Nove de Julho - UNINOVE \\ São Paulo, SP - Brasil \\ alinejgrocha@gmail.com \\ Marcos Rogério Mazieri \\ Doutor em Administração \\ Universidade Nove de Julho - UNINOVE \\ São Paulo, SP - Brasil \\ marcosmazzzieri@gmail.com
}

\begin{abstract}
Resumo: A constante pressão do mercado pela competitividade promove a necessidade do aumento da qualidade das entregas e diminuição de desperdícios e retrabalhos, utilizando equipes de alto desempenho e mantendo seus stakeholders cada vez mais satisfeitos. Por isso, foram aplicadas técnicas scrum-ágeis em um modelo de gestão de projetos de engenharia, baseado no modelo cascata. Portanto, este estudo tem por objetivo propor e aplicar técnicas scrum-ágeis no modelo de gestão de projetos de engenharia, que resultarão em um modelo híbrido, voltado a minimizar os impactos negativos presentes nas mudanças, observando o resultado refletido na equipe de gestão projetos de engenharia e nos stakeholders. A intervenção ocorreu por meio de pesquisa-ação em decorrência do papel de consultor realizado pelos autores, que identificaram os problemas e desenvolveram e implantaram as soluções. Foram verificadas redução no percentual de atrasos e melhoria dos indicadores de desempenho da empresa junto ao cliente. Além disso, foi realizada a revisão de um processo de desenvolvimento de projetos de engenharia já existente, tornando-o híbrido e propondo, dessa forma, uma nova forma de trabalho para as empresas de engenharia.
\end{abstract}

Palavras-chave: Projeto. Engenharia. Hibridismo. Ágil. Waterfall.

Abstract: The constant pressure from the market for competitiveness promotes the need to increase the quality of deliveries and reduce waste and rework, using high-performance teams and keeping its stakeholders more and more satisfied. Therefore, scrum-agile techniques were applied to an engineering project management model based on the waterfall model. Therefore, this study aims to propose and apply scrum-agile techniques, in the engineering project management model resulting in a hybrid model, minimizing the negative impacts present in the changes, observing the result reflected in the engineering project management team and stakeholders. The intervention took place through action research due to the role of consultant performed by the authors, in the identification of problems, development and implementation of solutions. There was a reduction in the percentage of delays and an improvement in the company's performance indicators with the customer. In addition, a review of an existing engineering project development process was carried out, making it hybrid, thus proposing a new way of working for engineering companies.

Keywords: Project. Engineering. Hybridity. Agile. Waterfall.

\section{Cite como}

American Psychological Association (APA)

Chies, T. G., Rocha, A. J. G., \& Mazieri, M. R. (2021, jan./jun.). Aplicação de hibridismo na gestão de equipes e stakeholders de múltiplos projetos de engenharia. Revista Inovação, Projetos e Tecnologias - IPTEC, São Paulo, 9(1), 60-75.

https://doi.org/10.5585/iptec.v9i1.18725. 


\section{Introdução}

A construção civil se caracteriza como uma atividade econômica representativa para um país (Uniemp, 2010). Em um cenário de competitividade de mercado, as empresas precisam ser dinâmicas e utilizar recursos profissionais e estratégicos em equipes de alto desempenho, que permitam tomadas de decisões oportunas e eficazes (Nascimento \& Santos, 2003), almejando a satisfação de seus clientes (Alencar \& Santana, 2010). No entanto, os resultados da falta de uma metodologia de gerenciamento de projetos eficiente são conhecidos pelo mercado da construção civil, tais como: obras atrasadas, estouros do orçamento dos projetos, equipes, clientes, construtores insatisfeitos, entre outros (Frej \& Alencar, 2010; Alencar \& Santana, 2010). Com o objetivo de gerenciar os custos, empresas do setor da construção civil tendem a lidar com as adversidades no dia a dia do gerenciamento de seus projetos, remetendo-se à metodologia waterfall (Vieira, 2002; Alencar \& Santana, 2010; Kern \& Formoso, 2006).

A metodologia de gestão de projetos, baseada em waterfall, objetiva proporcionar processos estruturados para auxiliar na resolução das principais lacunas de gerenciamento de projetos encontradas em uma empresa de construção civil, como a gestão de conflitos, gestão da equipe do projeto, gestão de stakeholders, capacitação do gerente de projetos, gestão de recursos, gestão de integração, gestão de comunicação e gestão de alterações de escopo juntamente com os impactos das mudanças ao longo do ciclo de vida dos projetos (Vieira, 2002; Kern \& Formoso, 2006; Alencar \& Santana, 2010). Considera-se estes os principais obstáculos na obtenção de sucesso de projetos nesse setor (Carvalho \& Rabechini, 2019).

De forma alternativa à metodologia waterfall, a metodologia ágil, utilizada principalmente para projetos inovadores, é caracterizada por projetos sem escopo definido, no qual o propósito é gerar valor aos stakeholders (Fernandez \& Fernandez, 2008; Cohen, Lindvall, \& Costa, 2004). Dado o atual cenário de cultura da inovação, algumas técnicas utilizadas pela abordagem ágil como: Daily Meeting, Sprint Planning, Sessões de Ideação e adoção da figura do PO (Product Owner) são aplicadas em conjunto com times multidisciplinares, para melhor especificar os planos de entregas e prototipação rápida de ideias de novos produtos e soluções (Cohen et al., 2004). Esses planos de entregas, associados às mudanças, são adaptados conforme as necessidades do negócio são descobertas (Schwaber \& Beedle, 2002).

Mudanças em um projeto são significativas para a gestão de tempo e custos na construção civil, porém empresas desse setor demonstram dificuldades em resolver esse problema de forma eficiente (Yap, Abdul-Rahman,Wang, \& Skitmore, 2017). Com base em 
Yap et al. (2012), pode-se constatar que a diretriz de gestão waterfall é observada no setor da construção civil, mesmo com os problemas recorrentes.

$\mathrm{Na}$ intervenção tratada neste relato técnico, foi analisada uma situação-problema que ocorre em uma unidade de negócio de uma organização que trabalha com gerenciamento de projetos. Essa unidade tem o foco em gerenciamento de obras para clientes do ramo varejista, não atuando na construção de edificações. Em função do alto número de solicitações de mudanças pelo cliente, há inúmeras revisões de projetos de engenharia, que são resultado da deficiência na comunicação, atrasos constantes nas entregas, falhas de qualidade e retrabalhos e, consequentemente, desperdícios de recursos humanos e elevação dos custos. Logo, foi levado em consideração, o desempenho do processo de gestão de projetos de engenharia (chamados de produtos) em uma equipe composta por profissionais de arquitetura e engenharia. Esse relato, portanto, propõe um artefato que conta com práticas advindas de metodologias ágeis para buscar eficiência no desenvolvimento dos produtos voltados ao campo de gerenciamento de mudanças. Além disso, os resultados da aplicação das práticas ágeis serão evidenciados, utilizando conceitos de equipes e stakeholders.

Dessa forma, o problema do relato será a resolução da seguinte questão: Como um modelo híbrido irá auxiliar na elevação do desempenho no processo de gestão de projetos de engenharia, em uma equipe de múltiplos projetos, considerando as intervenções scrum-ágil em um processo waterfall, para uma empresa de gerenciamento de obras de varejo? Dada a utilização da metodologia scrum-ágil, principalmente, em projetos de Tecnologia da Informação (TI), serão aplicadas essas técnicas de forma substantiva em projetos observados na organização em questão. A abordagem será qualitativa, utilizando o método de pesquisaação em função da proximidade e envolvimento dos pesquisadores na observação e resolução do problema.

\section{Referencial teórico}

As empresas buscam o gerenciamento de projetos como uma forma de alcançar melhores resultados. Em empresas da construção civil, geralmente são encontradas as abordagens tradicionais de gestão de projetos, como a waterfall (Vieira, 2002; Alencar \& Santana, 2010; Kern \& Formoso, 2006) mas, em pequena escala (Frej \& Alencar, 2010). Grande parte das empresas desse setor vêm enfrentando dificuldades em função da não adoção de formas de gestão que sejam eficazes em seus empreendimentos (Frej \& Alencar, 2010). Alguns dos resultados da falta de metodologia de gerenciamento de projetos do setor da construção 
civil são por exemplo: equipes de projetos desmotivadas, atraso de obras, projetos concluídos acima do orçamento e stakeholders insatisfeitos.

Ainda sobre as metodologias baseadas em waterfall, Carvalho e Rabechini (2019) abordam como característica, a gestão de alterações de escopo, que envolve um sistema de controle de mudanças e tem como objetivo a avaliação pelo gerente de projetos do impacto das alterações de escopo ao longo do ciclo de vida do projeto. Os impactos podem ser, segundo Kerzner (2011), aumento no orçamento ou alongamento do cronograma. Nesse contexto, Carvalho e Rabechini (2019) tratam que as mudanças ocorrem com maior frequência em projetos nos quais o escopo não foi bem planejado ou a gestão de stakeholders não foi exaustiva, ou ainda, que a avaliação dos riscos inerentes ao projeto não tenha sido desenhada adequadamente. Assim, entende-se que a abordagem de gestão de projetos tradicional visa a reduzir o número de mudanças com o propósito de redução de seus impactos.

De acordo com Fernandez e Fernandez (2008), considerando o cenário de mudanças nas empresas, são levantados questionamentos acerca das adaptações nas metodologias existentes e, assim, uma nova teoria de gerenciamento de projetos seria passível de avaliação. Embora a abordagem tradicional ao gerenciamento de projetos enfatize a robustez como vantagem, na qual os mesmos métodos e técnicas seriam aplicados a todos os projetos, um número crescente de autores ressalta o fato de que "um único tamanho não serve para todos" (Spundak, 2014).

Segundo Olsson, Sorensen e Leikvam (2015), os métodos ágeis podem ser vistos como uma reação aos métodos tradicionais ou plan-based, assumindo que existem soluções imprevisíveis para cada problema. Os autores consideram ainda que processos ágeis têm por objetivo responder às mudanças de requisitos sem retrabalho excessivo. Dentre os modelos de gerenciamento de projeto dessa linha, há o Scrum-ágil. Para Koskella e Howell (2002), o Scrum se desvia da doutrina convencional de gerenciamento de projetos em relação à falta de estrutura analítica do projeto e à descentralização das decisões.

Segundo Cohen et al., (2004), os projetos que utilizam a metodologia Scrum são divididos entre iterações (sprints) durante o seu ciclo de vida: (1) no pre-sprint planning, são selecionadas as tarefas contidas no backlog (total de atividades a serem executadas) para comporem o backlog da sprint; (2) durante a sprint, são selecionadas para a execução as atividades que compõem o backlog da sprint, havendo reuniões diárias para que ocorra o cumprimento das atividades da sprint, e (3) a reunião pós sprint, que analisa o progresso do projeto.

Além disso, o Scrum adota um processo do aprendizado empírico, assumindo que o conhecimento provém da experiência, sendo estruturado em três pilares, conforme exposto por 
Cohen et al. (2004) e Carvalho e Rabechini (2019): (1) transparência (todos envolvidos conseguem ver e compreender o que está ocorrendo), (2) inspeção (acompanha o produto sendo criado e o processo de criação) e (3) adaptação (conforme necessário, adaptar o produto ou o processo de criação). Sendo assim, cabe ressaltar que os modelos ágeis são mais indicados para projetos com maior incidência de mudanças e incertezas.

Dessa forma, é necessário haver a compreensão de que no scrum-ágil há requisitos iniciais que podem ser insuficientes e/ou ser modificados ao longo do ciclo de vida de um projeto, especialmente em projetos da área de TI. De acordo com Olsson et al. (2015), ao aplicar métodos ágeis fora da área de TI, é necessário visualizá-los mais como uma mudança de mindset do que em relação à aplicação de uma metodologia específica. Ainda segundo os autores, a aplicação de métodos ágeis no setor da construção civil inclui o aspecto iterativo e a busca por melhorias no valor de um projeto.

Complementarmente, segundo Melo (2016), a aplicação de métodos ágeis em projetos tem a capacidade de melhorar o desempenho dos projetos no setor de construção civil. No entanto, o autor ainda trata de barreiras para implantação da abordagem ágil em projetos desse setor, como por exemplo, as grandes somas de dinheiro e de tempo aplicadas neles. Nessa linha, ao comparar a construção de uma edificação com a fase antecessora, que é a elaboração de projetos de engenharia, esta possui menor custo envolvido e maior flexibilidade, além disso, tem maior influência na concepção e direção do projeto (Gould \& Joyce, 2009).

Nesse sentido, Minto (2002) coloca que um processo sequencial de desenvolvimento de projetos de engenharia, juntamente os modelos rígidos de planejamento, são incompatíveis com o chamado "caos" do processo criativo e intelectual de designs. No entanto, pôde-se verificar, em seu modelo genérico para organização do processo de projeto de engenharia, a base plandriving, sugerindo uma interatividade dentro das fases destacadas, conforme Figura 1. 
Figura 1 - Modelo genérico para organização do processo de projeto de forma integrada e simultânea

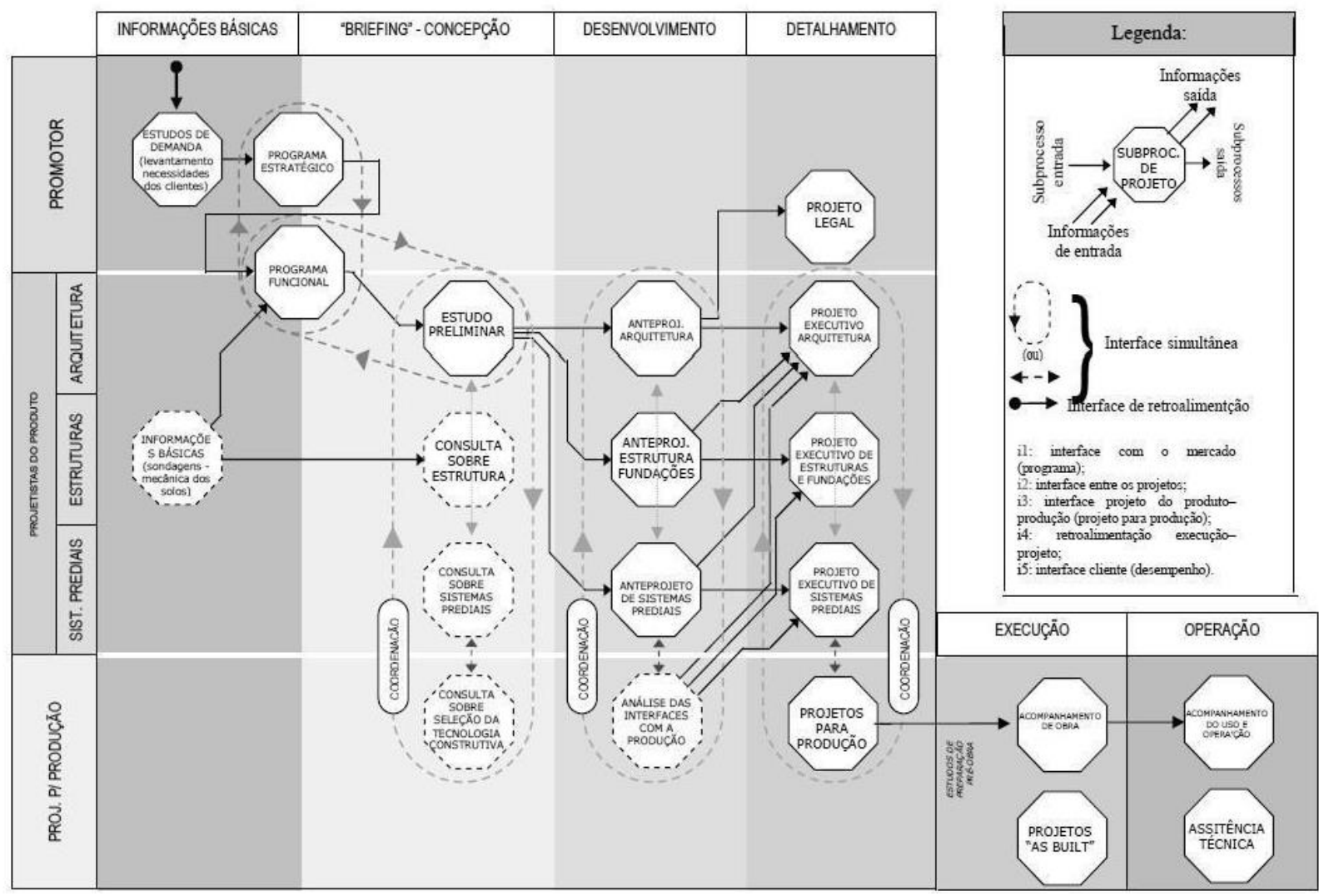

Fonte: Minto, 2002.

No modelo de Minto (2002), há a interação entre as disciplinas necessárias à construção como as disciplinas de Arquitetura, Estrutura e Sistemas Prediais, que é reforçada pela Associação Brasileira dos Escritórios de Arquitetura (Asbea, 2000). Segundo Melhado, Barros e Souza (1996), a interação entre disciplinas e as mudanças no gerenciamento de projetos de engenharia tendem a diminuir conforme o projeto vai sendo desenvolvido e detalhado para a execução da obra. Ou seja, a intervenção dos projetistas na concepção das soluções reduz nas fases finais de um projeto de engenharia. As fases iniciais são destinadas à concepção de ideias, na qual ocorre o processo criativo, que é conflitante com modelos rígidos de planejamento (Melhado et al., 1996; Minto, 2002).

Um exemplo de modelo flexível de planejamento, voltado para a abordagem ágil é o Minimum Viable Product ou Produto Mínimo Viável (MVP). Segundo o estudo de Lenarduzzi e Taibi (2016), o MVP é uma versão de um novo produto, desenvolvido com recursos mínimos, permitindo haver um aprendizado substancial pela equipe sobre os clientes com esforço mínimo. Além disso, o MVP é um processo iterativo de geração de ideias, que envolve prototipagem, apresentações, coleta de dados, análises e aprendizagem, sendo foco das 
atividades de desenvolvimento de negócios e produtos nas startups voltadas ao mercado de softwares (Lenarduzzi \& Taibi, 2016; Duc \& Abrahamsson, 2016).

Ao considerar que o MVP permite uma gestão de mudanças em projetos de TI, seria necessário aplicá-lo de forma substantiva em projetos de construção civil. No entanto, Ribeiro e Fernandes (2010) afirmam que a margem permitida para alterações do projeto de construção é pequena, pois mudanças mal gerenciadas no escopo de um projeto de engenharia causa impactos negativos. Uma abordagem híbrida de gerenciamento de projetos é proposta, portanto, para maximizar as oportunidades e reduzir os impactos.

Spundak (2014) afirma que, em relação ao hibridismo na gestão de projetos, ambas as abordagens tradicionais e ágeis têm suas vantagens e desvantagens, se comparadas a diferentes características do projeto. Ou seja, sendo possível realizar intervenções em etapas do processo de gestão de projetos, flexibilizando-o conforme a necessidade. Em relação ao uso da abordagem híbrida na prática, para Souza, Nery e Maccari (2017), a flexibilidade de escolhas e aplicação de abordagem híbrida mantêm o projeto adaptado às suas características e às necessidades do ambiente, além de gerar aprendizagem aos membros das equipes, favorecendo o desempenho ao iniciarem novos projetos.

Em relação aos stakeholders, há uma dificuldade de envolver o cliente, conforme premissa dos processos ágeis, uma vez que, de acordo com Ribeiro et al. (2010), torna-se difícil aos gerentes de projeto alavancar a experiência e o conhecimento das partes interessadas para que sejam elaborados e postos em prática planos de ação coerentes com as necessidades dos stakeholders, por meio da cooperação. Rabechini e Carvalho (2005) afirmam que a equipe é motivada e empenhada em alcançar o sucesso do projeto ao fazer interface com os stakeholders, sendo tanto as partes interessadas externas, quanto na própria corporação. Além disso, Thanhaim (2012) trata sobre a liderança da equipe que irá coordenar a interação complexa entre pessoas de diferentes habilidades e outras organizações, podendo tratar-se também de stakeholders. Thanhaim (2012) ainda aborda sobre a relação entre liderança gerencial e o desempenho da equipe, em que os gerentes de projeto devem promover um ambiente de trabalho que suporte os membros da equipe, promovendo uma satisfação com o trabalho, bom espírito de equipe, realizações, reconhecimentos, além de oportunidades para desenvolvimento, cultura de apoio e melhoria contínua, objetivando alcançar o sucesso nos projetos.

Logo, pode-se aferir a relevância da equipe, juntamente do gerente de projetos e dos stakeholders na gestão do projeto, almejando o sucesso. Em relação às abordagens waterfall e scrum-ágil, devem ser mapeadas as solicitações de mudança para que as tomadas de decisões do gerente de projetos sejam assertivas. Portanto, o relato irá identificar as técnicas scrum-ágeis, 
a serem aplicadas no modelo de gestão de projetos de engenharia proposto por Minto (2002), resultando em um modelo híbrido, voltado a minimizar os impactos negativos presentes nas mudanças, observando o resultado refletido na equipe de gestão projetos de engenharia e nos stakeholders.

\section{Método de produção técnica}

Este relato técnico trata da implantação de um modelo híbrido com o intuito de elevar o desempenho do processo gestão de projetos de engenharia (ou designs). Pode-se considerar a pesquisa-ação como abordagem metodológica, devido ao envolvimento de um dos autores na resolução do problema, sob o papel de consultor.

A pesquisa-ação de base empírica e abordagem qualitativa é apresentada na investigação de grupos ou coletividades de pequeno ou médio porte, na qual os pesquisadores e os participantes representativos de determinada situação se envolvem em uma ação ou na resolução de um problema coletivo, de forma cooperativa ou participativa (Thiollent, 2009; Thiollent, 2011). Esse tipo de metodologia de pesquisa permite a elevação do pesquisador ao papel de consultor, na identificação dos problemas, no desenvolvimento e implantação das soluções (Nunes \& Infante, 1996). O objetivo, além de responder a demandas, é construir novos conhecimentos, criticando a situação atual e propondo possíveis cursos de ação e estratégias (Thiollent, 2011).

Assim, o método aplicado foi baseado em Souza, Nery e Maccari (2017), que seguiram um roteiro de atividades em que foi permitida a compreensão da situação atual e identificação da abordagem waterfall do processo de desenvolvimento dos Projetos de Engenharia, além da proposição de soluções e estratégias que nortearam a implantação de um modelo híbrido no processo em questão, conforme demonstrado na Figura 2. 
Figura 2 - Fluxo de atividades (método adotado)

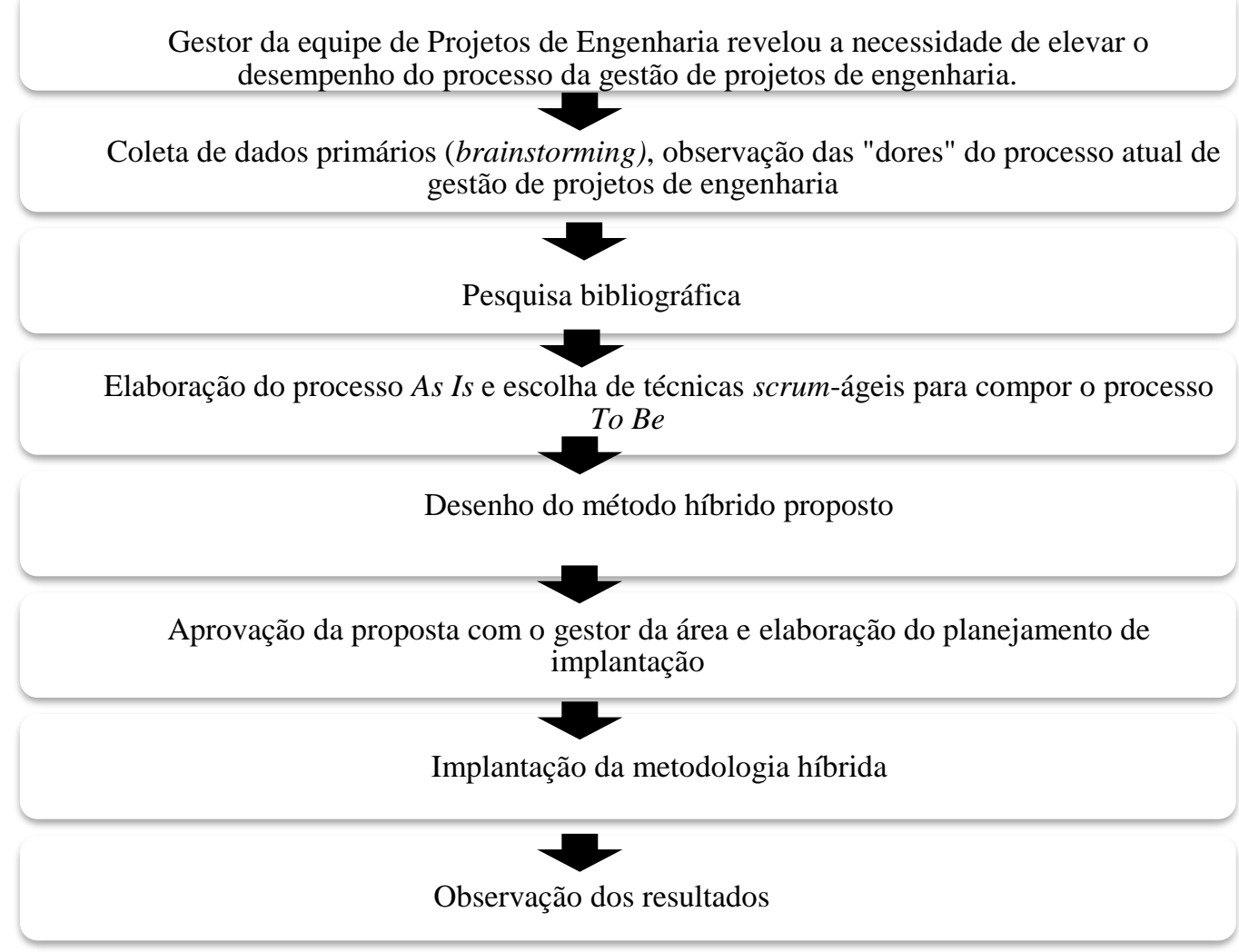

Fonte: Desenvolvido pelos autores.

Após o anúncio da necessidade de elevação do desempenho, uma das pesquisadoras, mesmo não trabalhando nesse setor dentro da organização, recebeu a demanda a título de consultoria. Como a pesquisa-ação considera variados tipos e métodos para coleta de dados, (Coughlan \& Brannick, 2005), foi realizada uma reunião de brainstorming com dois representantes do setor de projetos de engenharia para a coleta de dados primários, a fim de compreender o processo atual e avaliar os principais problemas que o setor enfrentava.

Complementarmente, a pesquisa-ação requer expressivo conhecimento prévio do ambiente corporativo da estrutura e dinâmica dos sistemas operacionais e dos conceitos teóricos que suportam tais sistemas (Coughlan \& Brannick, 2005). Assim, realizou-se pesquisa bibliográfica e, mediante a revisão teórica e o contexto do setor de projetos de engenharia da referida organização, entendeu-se que era necessário adotar uma abordagem híbrida. Foi então, confeccionado o desenho do processo de gestão de projetos de engenharia As Is, e elencadas as técnicas ágeis para propor o modelo To $B e$.

Após a confecção do modelo híbrido $T o B e$, foi realizada reunião com o gestor do setor, juntamente, com os líderes do setor para aprovação das técnicas e, por consequência, do modelo. A partir disso, traçou-se o plano de implantação em três ondas. 


\section{Aplicação prática}

A situação-problema ocorre em uma unidade de negócio de uma organização que trabalha com gerenciamento de projetos. Essa unidade de negócio tem o foco em gerenciamento de obras para clientes do ramo varejista, sendo que não atua construção de edificações. $\mathrm{O}$ escopo de prestação de serviço para o cliente em questão compreende serviços desde o levantamento físico no local da demanda solicitada por ele, perpassando pela fase de projeto de engenharia, que é a fase estudada neste relato, até a entrega da obra.

O objeto de estudo e a unidade de análise deste relato técnico são o modelo/processo de gestão de projetos de engenharia. A equipe, composta por aproximadamente 150 profissionais, desempenha os trabalhos dessa fase elaborando e/ou gerenciando múltiplos projetos, com o objetivo de entregar os produtos chamados de projetos de arquitetura, projetos complementares e demais documentos técnicos.

Os projetos de engenharia são terceirizados, em quase sua totalidade, e os colaboradores da equipe em questão atuam no gerenciamento dos terceiros, controle de qualidade e elaboração de revisões dos projetos recebidos pelos terceiros. Normalmente, há aproximadamente 200 projetos acontecendo de forma simultânea. A organização é matricial de forma projetizada para otimização de recursos, uma vez que ao longo do ano, há flutuações na quantidade de projetos em andamento. Essa equipe é caracterizada por uma interação incipiente dentre os gerentes de projetos das diversas disciplinas como arquitetura, estrutura, instalações e legalizações. Além disso, o controle de escopo do produto e o controle das mudanças são realizados individualmente por cada gerente de projeto, utilizando seus respectivos conhecimentos tácitos, com a supervisão do líder de projetos.

Com relação à percepção dos stakeholders, leva-se em consideração o setor de engenharia do cliente que se encontra descontente, relacionando os projetos desenvolvidos ao insucesso. Há um alto número de solicitações de mudanças efetuadas pelo cliente, que são, geralmente, em função do não atendimento ao programa de necessidades fornecido por ele e a não compreensão dos interesses e preocupações das partes interessadas.

Como consequência do elevado número de revisões e mudanças nos projetos de engenharia, há atrasos constantes nas entregas, falhas de qualidade e retrabalhos, resultando em desperdícios de recursos humanos, alta incidência de horas extras dos colaboradores com elevação dos custos, além da aplicação de multas contratuais. Assim, somada aos feedbacks negativos fornecidos pelo cliente, a falta de rotina de avaliações com feedbacks dentro da 
organização junto aos seus colaboradores e a alta pressão por entregas resultam na desmotivação da equipe de projetos de engenharia.

Além disso, são realizadas reuniões mensais com o vice-presidente da engenharia do cliente, em que são demonstrados indicadores de performance da organização em questão. Em março de 2019, antes do início das intervenções, o percentual de atrasos era de 16,5\% sobre as entregas realizadas, cuja média histórica de 2018 era de aproximadamente 16\%. Além disso, há também o ranking das gerenciadoras onde a organização em questão encontra-se em último lugar.

\subsection{Tipo de intervenção e mecanismos adotados}

Mediante a revisão teórica e a coleta de dados, por meio de observação, sobre o processo de confecção de projetos de engenharia, desenhou-se o processo As Is, do gerenciamento de projetos de engenharia, conforme Figura 3. Ou seja, observa-se que foram eliminadas algumas etapas em função do alto volume de projetos em andamento e a baixa complexidade técnica.

Figura 3 - Modelo de Gestão de Projetos de Engenharia As Is, na Organização em estudo

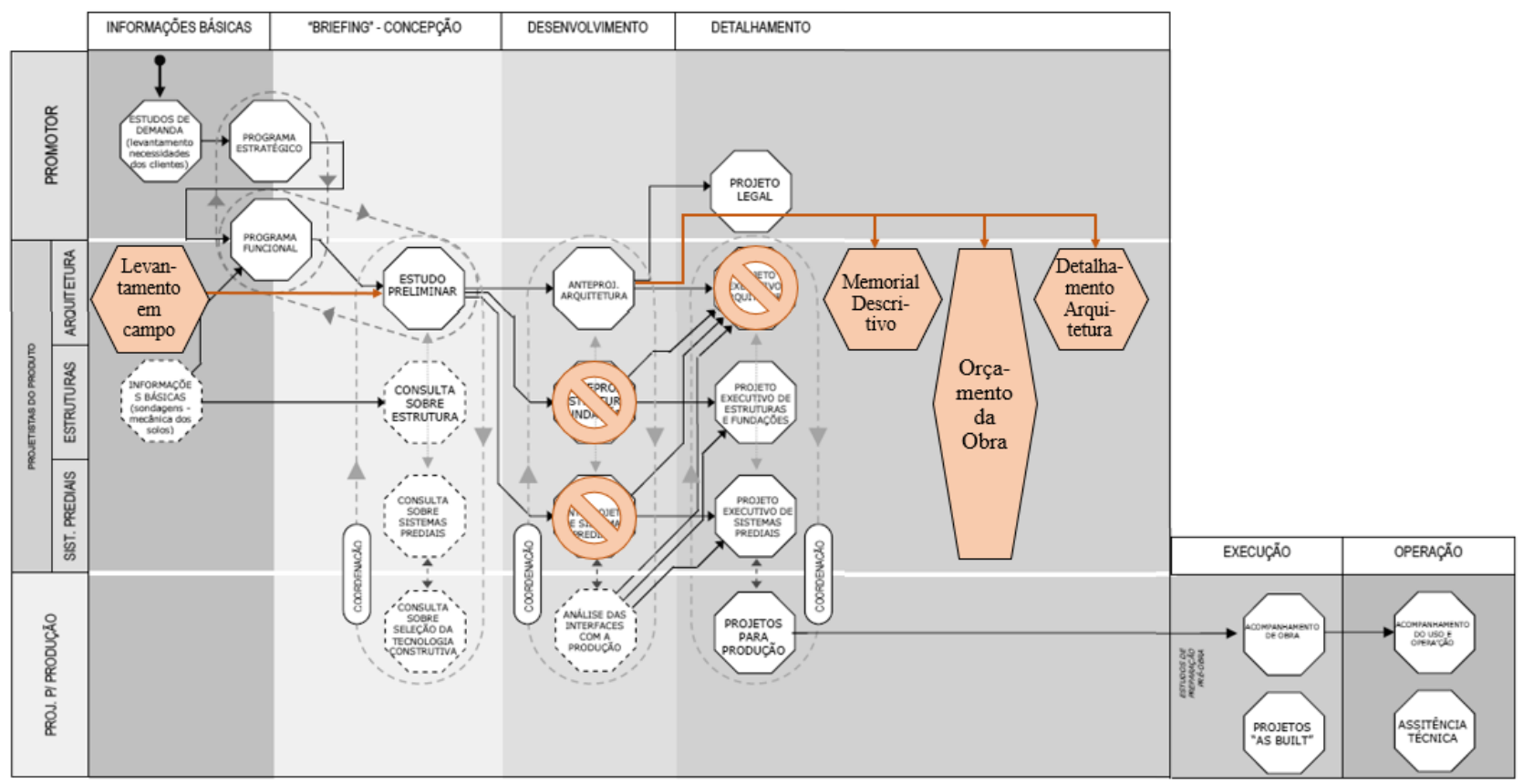

Fonte: Modelo de Minto (2002) adaptado pelos autores

Após o desenho do processo As Is, foram elencadas todas as dores do cliente e da equipe, de forma que o processo pudesse ser redesenhado para implantação de melhorias. Assim, estabeleceu-se o plano de implantação das melhorias em três ondas, que foi aprovado pelo gestor da área de projetos de engenharia: 
(1) Para atingir o problema de alto volume de mudanças, a primeira onda se caracteriza pela implantação do MVP em estágios iniciais do processo de desenvolvimento de projetos de engenharia. Para Melhado et al. (1996), conforme o projeto vai sendo desenvolvido e detalhado para a execução, as mudanças tendem a diminuir. A seguinte intervenção é contemplada na primeira onda: Reuniões periódicas, mais frequentes que as reuniões semanais (Semi-Daily Meetings) junto ao cliente (Product Owner), para apresentação de esboços dos layouts de arquitetura elaborados à mão (MVP). Nesse cenário, não são apresentados os desenhos finais, resultando em menos tempo e esforço na aprovação da concepção do projeto de arquitetura. A primeira onda iniciou em abril de 2019 e terminou em junho de 2019.

(2) Para o problema da falta de qualidade dos projetos de engenharia, a segunda onda se caracteriza pela implantação de sessões de ideação. Essas sessões são formadas por reuniões com equipes multidisciplinares (como por exemplo: arquitetura, estrutura, instalações, ar condicionado, obras, legalização) para analisar o layout aprovado pelo cliente e realizar brainstorming sobre novas ideias para a criação. Essa onda iniciou em junho de 2019, com uma fase de teste piloto em 30 projetos, porém, não foi iniciado em decorrência de repriorização da gerência do contrato.

(3) No direcionamento do problema relacionado aos atrasos constantes, a terceira onda se caracteriza pela implantação de reuniões diárias (Daily Meetings) com os gerentes de projeto para avaliar o status das entregas. Nesses encontros, são avaliadas as entregas com até dois dias de antecedência. Há um alto volume constante de demandas, aproximadamente 50 entregas/dia. As reuniões são divididas por grupos de profissionais, devido ao tamanho da equipe, e coordenadas pelos dois líderes de disciplinas: o de arquitetura e o de projetos complementares - esses líderes atuam nesse momento como Scrum Master, realizando a atualização de tarefas em To Do, Doing $e$ Done. Essa onda iniciaria em julho de 2019 em um piloto de 30 projetos.

\section{Resultados obtidos e análise}

As melhorias no processo promoveram a confecção do processo $T o B e$, cuja aprovação foi realizada em reunião com o gestor do setor, com os líderes de projetos de pesquisa e desenvolvimento, de arquitetura e o de projetos complementares. Uma vez aprovado o processo To $B e$, os futuros projetos iriam ser desenvolvidos levando em consideração as técnicas ágeis demonstradas no modelo, na Figura 4. 
Figura 4 - Modelo de Gestão de Projetos de Engenharia To Be, na Organização em estudo

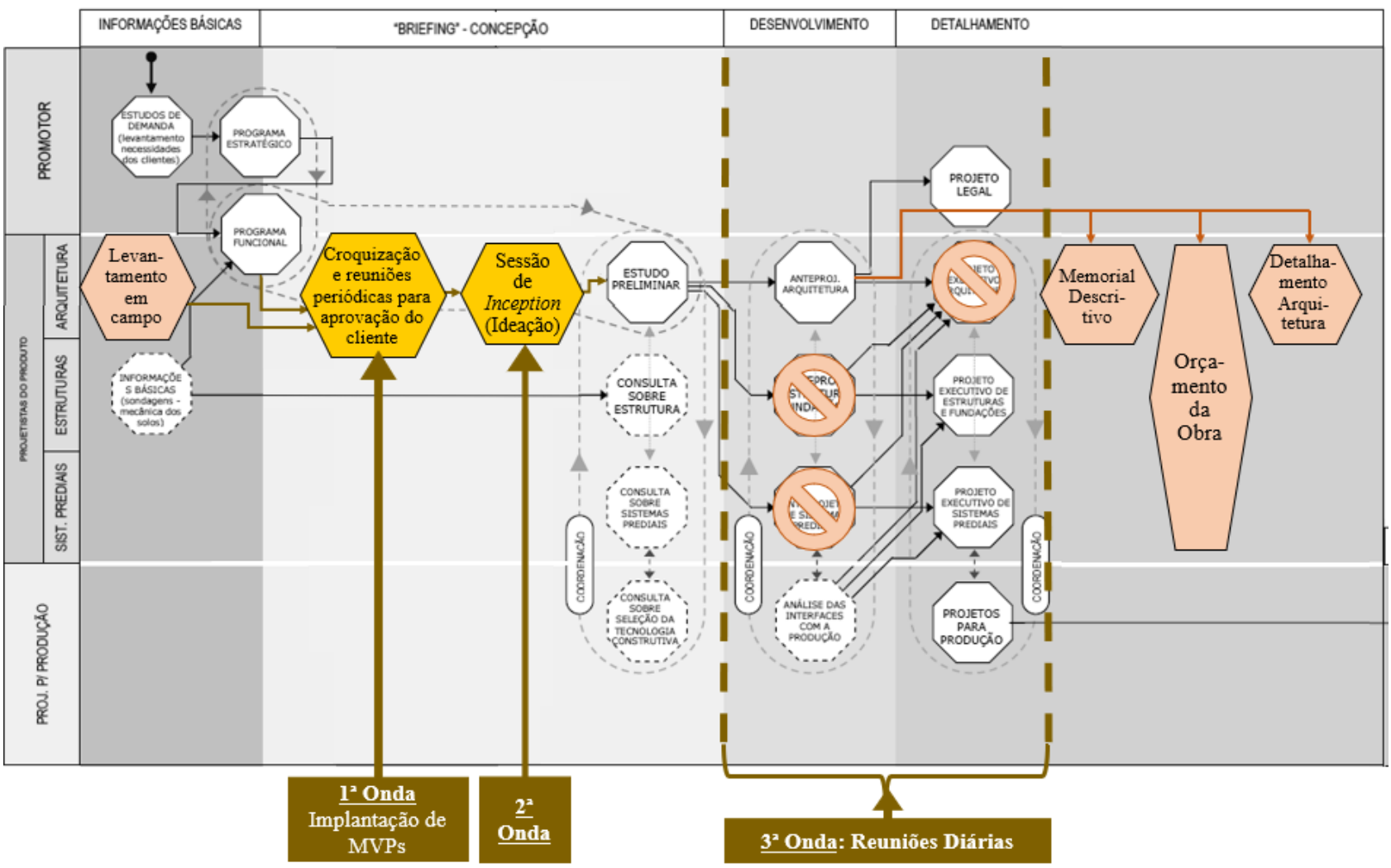

Fonte: Modelo de Minto (2002) adaptado pelos autores.

Em relação a segunda e terceira ondas, estas não foram iniciadas, portanto, seus resultados não serão contemplados neste relato. Quanto aos resultados da $1^{a}$ onda, o cliente, como stakeholder, reconheceu a melhoria no processo e informou que a gerenciadora estava entendendo, portanto, as necessidades do cliente. Além disso, houve a percepção pelos stakeholders da redução do número de mudanças nos designs. Em função da percepção positiva do cliente, a gerenciadora se beneficiou com o aumento do engajamento dos stakeholders.

Com a redução de feedbacks negativos por parte do cliente, houve um aceite generalizado por parte da equipe atuante nas intervenções, e o gestor da equipe de projetos de engenharia verificou, por meio de reuniões periódicas internas, que houve um incremento da motivação da equipe. Cabe ressaltar que ainda havia muitas tarefas a serem realizadas em conjunto com os recursos humanos $(\mathrm{RH})$ da unidade de negócio em questão, entretanto, o foco desta intervenção não é o objetivo deste relato.

Por fim, foi verificada quantitativamente uma redução no percentual de atrasos. Na apresentação dos indicadores de desempenho referentes ao mês de maio de 2019, o percentual de atrasos reduziu de $16,3 \%$ para $14 \%$ sobre as entregas realizadas. Isso significa que, mesmo que a $3^{\mathrm{a}}$ onda ainda não tivesse iniciado no momento da confecção deste relato, cujo foco é a 
redução dos atrasos, pôde-se verificar uma redução no percentual de atrasos, como reflexo da fase de MVP adotada.

\section{Conclusão}

A pesquisa-ação tem orientação voltada à mudança, sendo aplicável ao entendimento o planejamento e implementação delas (Coughlan \& Brannick, 2005). Dessa forma, com base no processo original de gestão de projetos de engenharia, denominado As Is, considerando a equipe de múltiplos projetos e os desafios presentes na empresa em questão, voltada para o gerenciamento de obras de varejo, foi proposto o processo To Be que incorporou intervenções scrum-ágil em um processo waterfall. A proposta das intervenções scrum-ágeis culminou em um processo de abordagem híbrida, considerando as três ondas de implantação de melhorias.

Após a operacionalização da primeira onda, houve elevação do desempenho no processo de gestão de projetos de engenharia e diminuição no percentual de atrasos, indicando uma prospecção positiva para as segunda e terceira ondas, em termos de resultados. Como consequência da redução dos atrasos, houve um melhor engajamento dos stakeholders, por entenderem a preocupação da empresa gerenciadora com suas necessidades.

Considerando a existência de particularidades em cada projeto, a utilização de metodologia híbrida de gestão de projetos é sugerida, uma vez que se pode adaptar a gestão às necessidades e mudanças. Spundak (2014) afirma que em relação ao hibridismo na gestão de projetos, ambas as abordagens tradicionais e ágeis têm suas vantagens e desvantagens, sendo possível adaptar o processo de forma a maximizar as oportunidades e minimizar os riscos da gestão do projeto.

Umas das limitações deste estudo foi o tempo reduzido para a efetivação das ações propostas, já que não houve a conclusão da implantação das ondas restantes. E, a implantação das ondas 2 e 3 serão propostas de estudos futuros, com o objetivo de tornar o processo To Be um artefato para aplicação prática, em contextos de múltiplos projetos de engenharia na construção civil.

\section{Referências}

Associação Brasileira dos Escritórios de Arquitetura - AsBEA, (2000). Manual de contratação dos serviços de arquitetura e urbanismo (2.ed.). São Paulo: Pini.

Alencar, L. H., \& Santana, M. O. (2010). Análise do gerenciamento de múltiplos projetos na construção civil. Revista Gestão e Projetos, 1 (1), 74-92. 
Biancolino, C. A., Kniess, C. T., Maccari, E. A., \& Rabechini Jr., R. (2012). Protocolo para Elaboração de Relatos de Produção Técnica. Revista Gestão e Projetos, 3(2), 294-307.

Carvalho, M. M., \& Rabechini Jr, R. (2019). Fundamentos em Gestão de Projetos.

Construindo Competências para Gerenciar Projetos (5.ed.). São Paulo: Editora Atlas.

Coghlan, D. \& Brannick, T. (2008). Doing action research in your own organization (2.ed.). London: Sage.

Cohen, D., Lindvall, M., \& Costa, P. (2004). An Introduction to Agile Methods. Advances in Computers. 62, 1-40.

Duc, A. N., \& Abrahamsson, P. (2016). Minimum Viable Product or Multiple Facet Product? The Role of MVP in Software Startups. In Sharp H., Hall T. (eds) Agile Processes, in Software Engineering, and Extreme Programming. Lecture Notes in Business Information Processing, vol 251. Springer, Cham.

Frej, T. A., \& Alencar, L. H., (2010). Fatores de sucesso no gerenciamento de múltiplos projetos na construção civil em Recife. Revista Produção, 20 (3), 322-334.

Fernandez, D. J. \& Fernandez, J. D. (2008). Agile Project Management - Agilism versus traditional approaches. Journal of Computer Information System, 49(2), 10-17.

Gould, F. E., \& Joyce, N. E. (2009). Construction project management. Prentice Hall.

Kern, A. P., Formoso, C. T. (2006). A Model for Integrating Cost Management and Production Planning and Control in Construction. Journal of Financial Management of Property and Construction, 11 (2), 75-90.

Kerzner, H. (2011). Gerenciamento de Projetos: uma abordagem sistêmica para planejamento, programação e controle. São Paulo: Editora Blücher.

Koskella, L., \& Howell, G., (2002). The Theory of project management: explanation to novel methods. Proceedings IGLC-10, Gramado, Brazil. 1-11.

Lenarduzzi, V., \& Taibi, D., (2016). MVP Explained: A Systematic Mapping Study on the Definitions of Minimal Viable Product (42th). Euromicro Conference on Software Engineering and Advanced Applications (SEAA).

Melhado, S. B. \& Barros, M. M. S.; Souza, A. L. R. (1996). Qualidade do projeto de edifícios: fluxogramas e planilhas de controle de projeto. São Paulo: Escola Politécnica/USP. (Documento CPqDCC n. 20091 - EP/SC-1).

Melo, M. (2016). Análise da abordagem ágil-enxuto no gerenciamento de projetos na indústria da construção civil. Anais do V SINGEP. São Paulo - SP - Brasil - 20, 21 e 22/11/2016.

Minto, F. M. (2002). Projeto Simultâneo na construção de edifícios. Tese Doutorado, Escola Politécnica, Universidade de São Paulo, São Paulo, Brasil. 
Nascimento, L. A. \& Santos, E. T. (2003). A indústria da construção na era da informação. Ambiente Construído, Revista da Antac, Porto Alegre, 3(1), 69-81.

Nunes, J. M., \& Infante, M. (1996). Pesquisa-ação: uma metodologia de consultoria. Rio de Janeiro: Editora FIOCRUZ. Available from Scielo Books.

Olsson, N. O. E., Sorensen, A. O., \& Leikvam, G. (2015). On the need for iterative real estate project models - Applying agile methods in real estate developments. Procedia Economics and Financ, 21, $524-531$.

Rabechini Jr., R.; Carvalho, M. M. (2005). Perfil das competências em equipes de projetos. Revista de Administração FGV.

Ribeiro, F. L., \& Fernandes, M. T. (2010). Exploring agile methods in construction small and medium enterprises: a case study. Journal of Enterprise Information Management. 23(2), $161-180$.

Schwaber, K., e Beedle, M. (2002). Agile Software Development with Scrum. Prentice-Hall, Upper Saddle River.

Souza, R. M. de, Nery, A. L. B., \& Maccari, E. A. (2017) Implantação de metodologia híbrida de gerenciamento de projetos: $\mathrm{O}$ caso de uma indústria metalúrgica familiar de médio porte. Anais do VI SINGEP - São Paulo - SP - Brasil - 13 e 14/11/2017.

Spundak, M. (2014). Mixed agile/traditional project management methodology - reality or illusion? Procedia - Social and Behavioral Sciences, 119, 939 - 948.

Thanhaim, H. (2012). The changing role of team leadership in multinational project environments. Revista Gestão e Projetos, 3 (2), 04-38.

Thiollent, M. (2009). Metodologia da pesquisa-ação. São Paulo: Cortez.

Thiollent, M. (2011). Action Research and Participatory Research: An Overview. International Journal of Action Research, 7(2), 160-174. Acesso em abril de 2019 https://nbn-resolving.org/urn:nbn:de:0168-ssoar-414079.

Uniemp (2010). Fórum Permanente das Relações Universidade-Empresa.

Vieira, M. M. (2002). PMI e a Construção Civil. PMI-RS Journal, n. 2, p. 10-13.

Yap, J. B. H., Abdul-Rahman, H., Wang, C., \& Skitmore, M. (2017). Exploring the underlying factors inducing design changes during building production. Production Planning \& Control : The Management of Operations, 29 (7), 586-601. 